\title{
Management of the antiphospholipid syndrome
}

\author{
Gerard Espinosa $・$ Ricard Cervera
}

Received: 15 February 2010 / Accepted: 04 March 2010

(c) Springer-Verlag 2010

\begin{abstract}
Antiphospholipid syndrome (APS) is characterized by recurrent venous or arterial thromboses, fetal losses and thrombocytopenia in the presence of antiphospholipid antibodies, namely lupus anticoagulant, anticardiolipin antibodies or antibodies directed to various proteins, mainly $\beta_{2}$ glycoprotein I, or all three. There is consensus in treating patients with APS and first venous thrombosis with oral anticoagulation to a target international normalized ratio (INR) of 2.0-3.0. A recent systematic review recommended a target INR of $>3.0$ in those patients with APS and arterial thrombosis. The approach in women with obstetric manifestations of APS is based on the use of aspirin plus heparin. The best treatment for patients with the catastrophic variant of the APS is a combination of anticoagulation, corticosteroids, and plasma exchange or intravenous immunoglobulins.
\end{abstract}

Keywords Antiphospholipid syndrome · Antiphospholipid antibodies · Therapy

G. Espinosa $\cdot$ R. Cervera (凶)

Department of Autoimmune Diseases, Institut Clínic de Medicina i Dermatologia, Hospital Clínic, Villarroel 170, 08036 Barcelona,

Catalonia, Spain

e-mail: rcervera@clinic.ub.es

\section{Introduction}

The antiphospholipid syndrome (APS) is characterized by recurrent venous or arterial thromboses, fetal losses and thrombocytopenia in the presence of antiphospholipid antibodies (aPL), namely lupus anticoagulant (LA), anticardiolipin antibodies (aCL) or antibodies directed to various proteins, mainly $\beta_{2}$ glycoprotein I ( $\left.\beta_{2} \mathrm{GPI}\right)$, or all three [1]. This syndrome was first recognized in patients with systemic lupus erythematosus (SLE) and later less frequently in those with other autoimmune disorders, but it is now well known that its development may also be independent of any underlying disease, and this form is termed "primary" APS [2].

Any combination of vascular occlusive events may occur in the same individual and the time between them also varies considerably from weeks to months or even years. Data from the largest series of patients with APS (the "Euro-phospholipid project" [3]) reveal that deep vein thrombosis, sometimes accompanied by pulmonary embolism, is the most frequent manifestation in this syndrome (reported in $38.9 \%$ of patients). Cerebrovascular accidents-either stroke (19.8\%) or transient ischaemic attacks (11.1\%) - are the most common arterial thrombotic manifestations. Early fetal loss (35.4\% of pregnancies), late fetal loss (16.9\% of pregnancies), and premature birth ( $10.6 \%$ of live births), and preeclampsia (9.5\% of pregnant females) are the most common fetal and obstetric complications. Thrombocytopenia (platelet count below $100,000 / \mu 1)$ appears in almost $30 \%$ of patients.

In 1992, Asherson [4] described the 'catastrophic' variant of APS as a condition characterized by multiple vascular occlusive events, usually affecting small vessels, presenting over a short period of time, and laboratory confirmation of the presence of aPL. Catastrophic APS 
represents less than $1 \%$ of APS cases [5]. However, patients with catastrophic APS usually end up in a lifethreatening situation with a mortality rate around $50 \%$ in the largest published series [6].

The purpose of this article is to review the treatment of thrombotic and obstetric manifestations of APS. In addition, we summarize the evidence-based information on the management of catastrophic APS.

\section{Primary prophylaxis of thrombosis in patients with antiphospholipid antibodies}

The therapeutic approach in asymptomatic carriers of aPL-that is, patients with SLE and aPL, patients with only obstetric manifestations of APS and healthy carriers of aPL but without previous thrombotic events-is still controversial. Present evidence-based knowledge does not support the widespread use of aspirin in all these aPL-positive patients. First, the annual thrombosis risk in asymptomatic aPL-positive patients ranges from $0 \%$ to $3.8 \%$ [7-11], being equivalent to that of major bleeding associated with the use of aspirin. Second, the only randomized clinical trial (APLASA study) in which 98 asymptomatic persistently aPL-positive individuals were randomized to receive a daily dose of $81 \mathrm{mg}$ of aspirin (48 patients) or placebo (50 patients) showed that these patients have a low overall annual incidence rate of acute thrombosis, and develop vascular events when additional thrombosis risk factors are present [12]. Specifically, the overall thrombosis incidence rate was 1.33 per 100 patient-years, that is 2.75 per 100 patient-years for aspirin-treated subjects and 0 per 100 patient-years for placebo-treated subjects (hazard ratio 1.04, 95\% confidence interval [CI] 0.69-1.56). All but one patient with thrombosis in either study group had concomitant thrombosis risk factors or systemic autoimmune disease at the time of thrombosis. Therefore, according to the results of this trial, asymptomatic, persistently aPL-positive individuals seem not to benefit from low-dose aspirin for primary thromboprophylaxis.

However, a more realistic approach with a lower degree of evidence would be to stratify these individuals according to some clinical features such as the presence of traditional congenital or acquired procoagulant risk factors, the aPL profile (persistently positive aCL and/or anti- $\beta_{2}$ GPI antibodies at moderate/high titres and/or unequivocally LA), and the coexistence of an underlying autoimmune disease (SLE in particular), to consider primary prophylactic therapy with low-dose aspirin (75-100 mg daily) [13]. In this sense, it is known that SLE represents a prothrombotic condition per se [14]. The disease itself acts as strong thrombophilic risk factor, primarily related to the chronic systemic inflammation and renal involvement. Furthermore, one study has shown that prophylactic aspirin should be given to all patients with SLE to prevent both arterial and venous thrombotic manifestations, especially in patients with aPL [15]. In the same study, the authors suggested that in selected patients with LA and a low bleeding risk, prophylactic oral anticoagulant therapy may provide higher utility. Therefore, there is currently consensus for primary thromboprophylaxis in these patients, mainly with low-dose aspirin.

An alternative to aspirin in SLE patients may be hydroxychloroquine. There is ample evidence for the protective role of this old drug against the development of both venous and arterial thrombosis $[16,17]$. These results may also open the door to the use of hydroxychloroquine in aPL-positive patients without SLE in the future.

Another interesting group of nonthrombotic patients with aPL is represented by women with obstetric morbidity, so-called obstetric APS. Two retrospective studies have shown that there is a low incidence of subsequent thrombosis after delivery in patients with APS treated with low-dose aspirin compared to untreated patients [18, 19]. These data suggest the beneficial role of aspirin in this subset of APS patients.

In addition, in all these groups of nonthrombotic aPLpositive subjects, cessation of oestrogen-containing oral contraceptive use, treatment of vascular risk factors if present, and the avoidance of smoking are all additional recommended therapeutic measures. Prophylaxis with heparin, administered subcutaneously, should certainly be given to cover high-risk situations, such as surgery.

More recently, an Italian collaborative study group has confirmed some of these recommendations [20]. They assessed the risk factors for a first thrombotic event in aPL-positive carriers and evaluated the efficacy of prophylactic treatments including low-dose aspirin and long-term warfarin or low-dose aspirin/heparin administered during high-risk periods (pregnancy/puerperium, immobilization and surgery). Long-term low-dose aspirin was initiated arbitrarily for at least one of the following reasons: SLE, other autoimmune disease, obstetric APS, or LA positivity. Multivariate logistic regression analysis showed that only hypertension and medium/high titres of IgG aCL were independent risk factors for a first thrombotic event in asymptomatic aPL carriers and, more interestingly, primary thromboprophylaxis was found to be protective.

\section{Secondary prophylaxis of thrombosis in patients with antiphospholipid syndrome}

Two metaanalysis have confirmed that the presence of aPL is related to an increased risk of thrombotic events. 
Specifically, this major risk is present both in patients with SLE [21] and in patients with primary APS [22]. Furthermore, a systematic review of published articles on APS has shown that LA is a clear risk factor for thrombosis, irrespective of the site and type of thrombosis, the presence of SLE and the methods used to detect them [23]. Moreover, high levels of aCL $[8,24]$ and concomitant positivity for anti- $\beta_{2}$ GPI and LA or aCL have been recognized as increasing the risk of thrombosis in patients with aPL [11]. In contrast, determining antiprothrombin antibodies does not seem to be helpful for defining a patient's risk of thrombosis [25].

The best secondary thromboprophylaxis in patients with definite APS, that is those who have suffered thrombosis and at least two positive determinations of aPL [1], is long-term anticoagulation [26]. This point is very important taking into consideration that some studies have included patients with only a single positive determination of aPL [27]. In other words, most patients included in some studies on secondary thromboprophylaxis did not have APS.

In accordance with a recent excellent systematic review [26], patients with definite APS with a first venous thrombosis should be treated with prolonged oral anticoagulation at a target international normalized ratio (INR) of 2.0-3.0 and those with an arterial event at an INR of 3.0-4.0. These conclusions are based on an analysis of nine cohort studies [9, 28-35], five subgroup analyses [36-39] and two randomized controlled studies [40, 41]. The main limitation of this review is the low-quality of some of the included studies (observational, nonrandomized, and retrospective cohorts). However, it is important to consider that despite the potential risks of missing information and reporting bias, they offer a more realistic picture of these patients.

One of the problems of high-intensity anticoagulation may be the higher risk of secondary bleeding, a point that the clinician has to consider at the time to decide the best treatment in these patients. In a study performed in 66 patients with definite APS with previous thrombosis treated with oral anticoagulation to a target INR of 3.5, the risk of intracranial and fatal bleeding was similar than in groups of patients treated to lower target ratios [33]. As a whole, the rate of major bleeding was six cases per 100 patientyears (95\% CI 1.6-15.0). The rate of intracranial bleeding was 1.5 cases per 100 patient-years (95\% CI, 0.04-8.4) and the rate of thrombotic recurrence was 9.1 cases per 100 patient-years (95\% CI, 3.3-19.6). Nevertheless, the systematic review found that repeated thromboses were more frequent and associated with a higher mortality than haemorrhagic complications in patients taking warfarin [26].

One important and novel aspect in the management of APS is that patients should be stratified and treated according to some clinical and immunological characteristics in addition to aPL positivity [1]. These factors are discussed in the following sections.

Thrombophilic risk factors

It is advisable to categorize APS patients according to the presence or absence of classic thrombophilic risk factors such as hypertension, diabetes mellitus, hypercholesterolaemia, or tobacco use, because they may contribute to modifications in the eventual risk factor profile [9]. Close control of these factors should be an important clue in the management of patients with APS and thrombosis.

The two most common genetic causes of thrombophilia are the Leiden mutation of factor $\mathrm{V}$ and the G20210A mutation of prothrombin. The prevalence of factor $\mathrm{V}$ Leiden varies between different populations, averaging $2 \%$ to $10 \%$ for the heterozygous form and $1.5 \%$ for the homozygous form [42]. Heterozygous factor V Leiden is present in approximately $20 \%$ of patients with a first thromboembolism and is homozygous in $2 \%$ of these patients [43]. Factor V Leiden increases the risk of an initial venous thromboembolism. For the heterozygotic and homozygotic forms of factor V Leiden, the relative risk (RR) is 3 to 10 and 79, respectively [44]. The prothrombin mutation G20210A increases the risk of an initial venous thromboembolism (RR 2-5) [45]. Its prevalence in the general population varies between different ethnic groups, averaging $1 \%$ to $5 \%$, but its prevalence in patients with an initial venous thromboembolism is around 9\% [46]. However, the role of inherited thrombophilia in the thrombotic risk of patients with APS is contradictory. In general, the prevalence of factor $\mathrm{V}$ Leiden and prothrombin gene mutation are similar in patients with APS and healthy individuals and their presence does not increase the risk of a thrombotic event [47]. However, Galli et al. [48] suggested that factor V Leiden is associated with the thrombotic risk in patients with LA. Regarding prothrombin mutation, its presence has not been related to increased thrombotic risk in patients with APS [48].

No studies have sought to determine whether thrombophilic defects are risk factors for recurrent venous thrombosis during anticoagulant therapy in patients with APS. Data not yet published from our cohort of patients shows that patients with inherited thrombophilic defects, such as factor $\mathrm{V}$ Leiden or prothrombin mutation, do not show an increased risk of recurrent thrombosis under anticoagulant therapy. Further studies are necessary to establish the exact role of these genetic thrombophilic defects in patients with APS. 
Profile of the antiphospholipid antibodies

The latest revised classification criteria recommend classifying these patients into different categories according to their aPL profile [1]. Patients with $\mathrm{LA}, \mathrm{IgG}$ aCL at high titres, or anti- $\beta_{2}$ GPI antibodies plus LA or aCL have the highest thrombotic risk [49]. There is no evidence regarding the effectiveness of more intensive therapy in these patients. However, common sense dictates the need for closer clinical and therapeutic monitoring to ensure a correct INR in patients with thrombosis and any of these immunological profiles.

Persistence of antiphospholipid antibody positivity

At present, there is no evidence regarding the usefulness of repeat aPL testing in patients who meet the criteria for APS. However, a recent prospective study in patients with SLE has demonstrated that LA-positive patients have a greatly increased risk of thrombosis, both at the arterial and venous level. Interestingly, LA-negative patients but with persistently positive aCL (defined as positive in more than two-thirds of the determinations) have an increased risk of thrombosis at the expense of arterial events, whereas in LA-negative and transiently aCL-positive patients (defined as positive on at least two occasions, but in fewer than two-thirds of the determinations), the risk of thrombosis, both arterial and venous, is no different from that in aPL-negative SLE patients [50]. Similar results have been obtained by our group in patients with APS [51]. The adjusted risk for recurrent thrombosis during follow-up is increased in persistently positive aPL patients (defined as more than $75 \%$ of the aPL determinations positive during follow-up) compared with transiently positive aPL patients. The profile of persistently positive aPL related to the appearance of thrombosis during follow-up is a combination of $\mathrm{IgG}$ aCL and LA. The role of high aCL titres ( $>40$ IgG or IgM phospholipid units), which is a laboratory criterion for APS classification, in the risk for thrombosis recurrence was not performed in these two studies.

Recurrent thrombotic events despite optimal anticoagulation

In the 5-year follow-up of the "Europhospholipid" cohort of 1,000 APS patients, recurrent thrombotic events appeared in 166 of them (16.6\%), and the most common were strokes $(2.4 \%)$, transient ischaemic attacks $(2.3 \%)$, deep-vein thromboses $(2.1 \%)$, and pulmonary embolism (2.1\%) [52]. The best evidence on which to base any therapeutic recommendation in this group of patients comes from a published systematic review of the literature [26]. Of the 180 recurrent thrombotic events reported, $49(27 \%)$ occurred in patients treated with warfarin. Within this group, the actual INR at the time of the event was $<3.0$ in 42 patients $(86 \%)$. However, a subtherapeutic INR at the time of thrombosis may only represent inadequate anticoagulation and not treatment failure. Recurrences were infrequent among patients effectively receiving oral anticoagulation at an INR of 3.0-4.0. Therefore, patients with APS with recurrent venous events should be treated with warfarin at an INR of $>3.0$. This recommendation is based on cohort studies because randomized controlled trials included few patients with this profile. In addition, there are no evidence-based data that allow additional antithrombotic treatment such as aspirin to be recommended for patients who experience recurrent events while receiving oral anticoagulants at an INR of $>3.0$. However, adding low-dose aspirin to oral anticoagulation may be a reasonable option in these patients.

Seronegative antiphospholipid syndrome and patients in whom the antiphospholipid antibody test turns negative

There are a small number of patients with the classical features of APS but with persistently negative aPL, leading to the concept of "seronegative" APS. First, careful differential diagnosis with other causes of thrombophilia and repeat testing are mandatory before this diagnosis can be made. Antibodies may be directed against other phospholipids such as phosphatidylethanolamine, phosphatidylinositol, or against components of the protein $\mathrm{C}$ pathway or annexin V. An aPL-negative conventional test result may also be caused by a "technical" problem in that the test cannot detect aPL. In this regard, in a study investigating the possibility of detecting aPL by immunostaining on thin-layer chromatography plates, aPL were detected in the serum from ten patients with features of APS but aPL-negative by ELISA [53].

Few patients with APS and previously positive for aPL may become negative over time. Erkan et al. [54] demonstrated that aPL remains stable in at least threequarters of subsequent tests, regardless of the laboratory performing the test. At present, the factors related to the "disappearance" of aPL are completely unknown. There is no scientific evidence regarding the increased thrombotic risk and the role of prophylactic treatment in these two groups of patients. Also, the question as to whether or not treatment should be stopped after (spontaneous) disappearance of aPL needs further study. However, in patients with seronegative APS and previous thrombotic events, common sense suggests the need for long-term 
anticoagulation. Although anticoagulation withdrawal may be safe in APS patients when aPL become negative [55], further evidence describing the clinical importance of aPL disappearance is needed before the formal recommendation of this approach.

Patients with antiphospholipid antibodies who do not display formal classification criteria for antiphospholipid syndrome

Although a set of classification criteria have been established [1], some patients present a diagnostic and therapeutic challenge. Examples of this are patients with thrombosis and repeated low titres of aCL $(<40 \mathrm{IgG}$ or IgM phospholipid units) or anti- $\beta_{2}$ GPI antibodies and negative LA, or patients with aPL and clinical features not included as clinical criteria, such as nonbacterial thrombotic endocarditis, seizures or nephropathy. In the first example, the diagnostic problem is due to the absence of data to establish the threshold between moderate/high levels and low levels. In the second case, the recently updated classification criteria for APS indicate that these clinical characteristics are frequently related to aPL. However, their inclusion as classification criteria for definite APS may decrease the diagnostic specificity [1]. In both cases, the therapeutic approach is similar. Long-term anticoagulation is advisable in an approach similar to that in patients who carry the laboratory and clinical criteria of APS.

\section{Management of obstetric antiphospholipid syndrome}

Good results with low-dose aspirin alone, with success rates over 70\%, have been achieved in APS patients with two or more pregnancy losses [56-62]. The daily doses of aspirin used in these studies ranged between $75 \mathrm{mg}$ and $100 \mathrm{mg}$. The optimal antiaggregant dose of aspirin is still uncertain. Although doses as high as $325 \mathrm{mg}$ three times daily have been used in the past, there is no evidence that doses higher than $75 \mathrm{mg}$ daily are more effective in preventing thrombotic events, whilst toxicity is probably dose-related [63].

The use of heparin is a logical approach to the treatment of a disorder resulting from thrombosis. In the earliest published case series in 1990 [64] it was observed that under heparin (mean dose 24, $700 \mathrm{U} /$ day), 14 of 15 pregnancies in 14 women with aPL and a history of 29 abnormal pregnancy outcomes including 28 miscarriages ended in live births. There is now accumulating experience with the use of low-molecular-weight heparins both in pregnant and nonpregnant patients for the prevention of complications associated with aPL and there is also evidence that low-molecular-weight heparins do not cross the placenta and they are safe and effective in pregnancy [65]. Low-molecular-weight heparins have potential advantages over unfractionated heparin during pregnancy because they cause less heparin-induced thrombocytopenia, have the potential for once-daily administration because of better bioavailability and longer half-life, and may result in a lower risk of heparin-induced osteoporosis [65].

The current most commonly recommended treatment for women with recurrent pregnancy losses and aPL is heparin and low-dose aspirin, starting therapy when the pregnancy is confirmed [66-70]. This recommendation is essentially based on three clinical trials which have shown better obstetric outcomes using aspirin plus heparin than aspirin alone [71-73]. Two of them were randomized trials [71, 73], whereas Kutteh [72] assigned treatment in a consecutive way, which limited the validity of the results. The results of the two studies [71,72] were quite similar. The live birth rate in the heparin-treated groups was higher than in women treated with aspirin alone. In contrast, Farquharson et al. [73] found similar results with lowdose aspirin alone or in combination with dalteparin. No differences were found between treatment groups with respect to obstetric complications. However, potential limitations of these studies need to be addressed. The study by Kutteh [72] excluded women with LA, whereas in the study by Rai et al. [71], 80\% of the patients had LA in the absence of aCL. This is a very infrequent profile as $\mathrm{aCL}$ are usually present in over $80 \%$ of patients with APS. Importantly, in both reports there is no mention as to whether patients had been treated with aspirin alone in a previous pregnancy ending in miscarriage. Most patients recruited for both studies had early pregnancy losses, a condition usually seen and treated by specialists dealing with fertility difficulties. It is thus plausible that APS referral centres are receiving patients who have failed in their first treatment attempt with low-dose aspirin, and in these patients therapy with heparin plus low-dose aspirin is recommended [70]. In the study by Rai et al. [71] most miscarriages occurred before 13 weeks of gestation and there was no difference between the two treatments in the outcomes of pregnancies that advanced beyond this time. Undoubtedly, aspirin at a daily dose of $100 \mathrm{mg}$ can be started when pregnancy is contemplated, but beginning heparin or prednisone prior to conception exposes the patient to an unknown duration of these drugs and potential risks. In the study by Rai et al. [71] aspirin treatment was started after diagnosis of pregnancy. In both studies $[71,72]$, the outcomes in the aspirin-only group were worse than those in other series with the same schedule $[56,58-61,74]$. 
Two studies, a double-blind randomized placebo-controlled trial [75] and a subgroup analysis of one randomized controlled trial performed by Cowchock and Reece [76], have not shown any benefit of adding aspirin to an intensive obstetric care and placebo treatment. The prognosis in both the aspirin and control groups was remarkably good, with success rates over $80 \%$. However, most patients included in these studies had only low-titre aCL [75] or a lack of significant adverse obstetric histories [76]. In addition, treatment was started when pregnancy was diagnosed or on discovery of aPL during pregnancy, but not before conception. Thus, these two studies emphasize a very important aspect in the management of these patients and the only one where general agreement is found: that they should undergo close fetal and maternal surveillance by a well-coordinated multidisciplinary team including obstetricians, internists/rheumatologists, and haematologists.

A recently published American College of Chest Physicians evidence-based clinical practice guidelines for the management of antithrombotic therapy in pregnancy recommend in women with recurrent early pregnancy loss or unexplained late pregnancy loss who test positive for aPL and have no history of venous or arterial thrombosis, the antepartum administration of prophylactic or intermediate-dose unfractionated heparin or prophylactic low molecular weight heparin combined with aspirin (grade 1B) [65].

\section{Management of catastrophic antiphospholipid syn- drome}

Catastrophic APS is an unusual form of presentation of APS that represents less than $1 \%$ of APS cases reported [3]. In the earliest published series, the mortality rate was approximately $50 \%[77,78]$. However, in a recent study by Bucciarelli et al. [6], the mortality rate had fallen by some $20 \%$. This is clearly due to the use as first-line therapy of anticoagulation, corticosteroids, plasma exchange, and intravenous immunoglobulins (IVIG).

The mechanisms involved in the causation and pathogenesis of catastrophic APS are not completely understood. It is still unclear why some patients develop recurrent thromboses, mainly affecting large vessels (classic APS), while others develop rapidly recurring vascular occlusions, predominantly affecting small vessels (catastrophic APS). A possible mechanism in the pathogenesis of catastrophic APS is the systemic inflammatory response syndrome (SIRS), which is presumed to be caused by excessive cytokine release from affected and necrotic tissues.

The higher recovery rate was achieved by a combination of anticoagulation, corticosteroids and plasma exchange (77.8\%), followed by a combination of anticoagulation, corticosteroids, plasma exchange and/or IVIG (69\%). In contrast, concomitant treatment with cyclophosphamide did not demonstrate additional benefit [6]. However, Bayraktar et al. [79] demonstrated that the use of cyclophosphamide improves survival in patients with catastrophic APS associated with SLE.

More interestingly, the mortality rate decreased from $53 \%$ in patients diagnosed before 2000 to $33.3 \%$ in those diagnosed from 2001 to February 2005 ( $p=0.005$; OR $2.25,95 \%$ CI 1.27-3.99) [6]. Patients in the second period were younger than those in the first $(34.4 \pm 11.8$ and $39.4 \pm 14.8$ years, respectively; $p=0.016$ ) and a higher number of precipitating factors for catastrophic APS episodes were identified in the second period. In addition, in the patients with catastrophic APS diagnosed from 2001, treatments including a combination of anticoagulation, corticosteroids, plasma exchange and/or IVIG were more frequently administered than in the earlier period $(28.6 \%$ vs $13.3 \%)$. We consider that the difference, although statistically significant, in the mean age at the time of catastrophic APS between patients in the first and second period was not high enough to explain the decrease in mortality rate in the second period. The higher number of precipitating factors identified in the second period may indicate that physicians are increasingly recognizing catastrophic APS and, therefore, earlier and more specific therapies for the precipitating factors as well as for the catastrophic event are prescribed. However, we consider that the main explanation for this significant reduction in mortality is the more frequent use of treatment with a combination of anticoagulation, corticosteroids, plasma exchange and/or IVIG. According to the results of this study, we therefore strongly advocate the use of combined treatment with anticoagulation, corticosteroids and plasma exchange as first-line therapy for patients with catastrophic APS [6]. This is in accordance with the international consensus guidelines for the management of catastrophic APS [80].

Conflict of interest statement The authors declare that they have no conflict of interest related to the publication of this article.

\section{References}

1. Miyakis S, Lockshin MD, Atsumi T et al (2006) International consensus statement on an update of the classification criteria for definite antiphospholipid syndrome (APS). J Thromb Haemost 4:295-306

2. Asherson RA, Khamashta MA, Ordi-Ros J et al (1989) The "primary" antiphospholipid syndrome: major clinical and serological features. Medicine (Baltimore) 68:366-374

3. Cervera R (2008) Lessons from the "Euro-Phospholipid" project. Autoimmun Rev 7:174-178 
4. Asherson RA (1992) The catastrophic antiphospholipid syndrome. J Rheumatol 19:508-512

5. Cervera R, Piette JC, Font J et al (2002) Antiphospholipid syndrome: clinical and immunologic manifestations and patterns of disease expression in a cohort of 1,000 patients. Arthritis Rheum 46:1019-1027

6. Bucciarelli S, Espinosa G, Cervera R et al (2006) Mortality in the catastrophic antiphospholipid syndrome: causes of death and prognostic factors in a series of 250 patients. Arthritis Rheum 54:2568-2576

7. Shah NM, Khamashta MA, Atsumi T et al (1998) Outcome of patients with anticardiolipin antibodies: a 10 year follow-up of 52 patients. Lupus 7:3-6

8. Finazzi G, Brancaccio V, Moia M et al (1996) Natural history and risk factors for thrombosis in 360 patients with antiphospholipid antibodies: a four-year prospective study from the Italian Registry. Am J Med 100:530-536

9. Girón-González JA, García del Rio E, Rodríguez C et al (2004) Antiphospholipid syndrome and asymptomatic carriers of antiphospholipid antibody: prospective analysis of 404 individuals. J Rheumatol 31:1560-1567

10. Somers E, Magder LS, Petri M (2002) Antiphospholipid antibodies and incidence of venous thrombosis in a cohort of patients with systemic lupus erythematosus. J Rheumatol 29:2531-2536

11. Forastiero R, Martinuzzo M, Pombo G et al (2005) A prospective study of antibodies to beta2-glycoprotein I and prothrombin, and risk of thrombosis. J Thromb Haemost 3:1231-1238

12. Erkan D, Harrison MJ, Levy R et al (2007) Aspirin for primary thrombosis prevention in the antiphospholipid syndrome: a randomized, double-blind, placebo-controlled trial in asymptomatic antiphospholipid antibody-positive individuals. Arthritis Rheum 56:2382-2391

13. Gerosa M, Chighizola C, Meroni PL (2008) Aspirin in asymptomatic patients with confirmed positivity of antiphospholipid antibodies? Yes (in some cases). Intern Emerg Med 3:201-203

14. Erkan D (2006) Lupus and thrombosis. J Rheumatol 33:1715-1717

15. Wahl DG, Bounameaux H, de Moerloose P et al (2000) Prophylactic antithrombotic therapy for patients with systemic lupus erythematosus with or without antiphospholipid antibodies: do the benefits outweigh the risks? A decision analysis. Arch Intern Med 160:2042-2048

16. Ruiz-Irastorza G, Egurbide MV, Pijoan JI et al (2006) Effect of antimalarials on thrombosis and survival in patients with systemic lupus erythematosus. Lupus 15:577-583

17. Erkan D, Yazici Y, Peterson MG et al (2002) A cross-sectional study of clinical thrombotic risk factors and preventive treatments in antiphospholipid syndrome. Rheumatology (Oxford) 41:924-929

18. Erkan D, Merrill JT, Yazici Y et al (2001) High thrombosis rate after fetal loss in antiphospholipid syndrome: effective prophylaxis with aspirin. Arthritis Rheum 44:1466-1467

19. Tincani A, Taglietti M, Biasini C et al (2002) Thromboembolic events after fetal loss in patients with antiphospholipid syndrome: comment on the article by Erkan et al. Arthritis Rheum 46:1126-1127

20. Ruffatti A, Del Ross T, Ciprian M et al (2009) Risk factors for a first thrombotic event in antiphospholipid antibody carriers. A multicenter retrospective follow-up study. Ann Rheum Dis 68:397-399

21. Wahl DG, Guillemin F, de Maistre E et al (1997) Risk for venous thrombosis related to antiphospholipid antibodies in systemic lupus erythematosus -a meta-analysis. Lupus 6:467-473

22. Wahl DG, Guillemin F, de Maistre E et al (1998) Meta-analysis of the risk of venous thrombosis in individuals with antiphospholipid antibodies without underlying autoimmune disease or previous thrombosis. Lupus 7:15-22

23. Galli M, Luciani D, Bertolini G et al (2003) Lupus anticoagulants are stronger risk factors for thrombosis than anticardiolipin anti- bodies in the antiphospholipid syndrome: a systematic review of the literature. Blood 101:1827-1832

24. Turiel M, Sarzi-Puttini P, Peretti R et al (2005) Thrombotic risk factors in primary antiphospholipid syndrome: a 5-year prospective study. Stroke 36:1490-1494

25. Galli M, Luciani D, Bertolini G et al (2003) Anti-beta 2-glycoprotein I, antiprothrombin antibodies, and the risk of thrombosis in the antiphospholipid syndrome. Blood 102:2717-2723

26. Ruiz-Irastorza G, Hunt BJ, Khamashta MA (2007) A systematic review of secondary thromboprophylaxis in patients with antiphospholipid antibodies. Arthritis Rheum 57:1487-1495

27. Lim W, Crowther MA, Eikelboom JW (2006) Management of antiphospholipid antibody syndrome: a systematic review. JAMA 295:1050-1057

28. Rosove MH, Brewer PM (1992) Antiphospholipid thrombosis: clinical course after the first thrombotic event in 70 patients. Ann Intern Med 117:303-308

29. Derksen RH, de Groot PG, Kater L et al (1993) Patients with antiphospholipid antibodies and venous thrombosis should receive long term anticoagulant treatment. Ann Rheum Dis 52:689-692

30. Khamashta MA, Cuadrado MJ, Mujic F et al (1995) The management of thrombosis in the antiphospholipid-antibody syndrome. N Engl J Med 332:993-997

31. Krnic-Barrie S, O'Connor CR, Looney SW et al (1997) A retrospective review of 61 patients with antiphospholipid syndrome. Analysis of factors influencing recurrent thrombosis. Arch Intern Med 157:2101-2108

32. Muñoz-Rodríguez FJ, Font J, Cervera R et al (1999) Clinical study and follow-up of 100 patients with the antiphospholipid syndrome. Semin Arthritis Rheum 29:182-190

33. Ruiz-Irastorza G, Khamashta MA, Hunt BJ et al (2002) Bleeding and recurrent thrombosis in definite antiphospholipid syndrome: analysis of a series of 66 patients treated with oral anticoagulation to a target international normalized ratio of 3.5. Arch Intern Med 162:1164-1169

34. Ames PR, Ciampa A, Margaglione M et al (2005) Bleeding and re-thrombosis in primary antiphospholipid syndrome on oral anticoagulation: an 8-year longitudinal comparison with mitral valve replacement and inherited thrombophilia. Thromb Haemost 93:694-699

35. Wittkowsky AK, Downing J, Blackburn J et al (2006) Warfarinrelated outcomes in patients with antiphospholipid antibody syndrome managed in an anticoagulation clinic. Thromb Haemost 96:137-141

36. Ginsberg JS, Wells PS, Brill-Edwards P et al (1995) Antiphospholipid antibodies and venous thromboembolism. Blood 86:3685-3691

37. Prandoni P, Simioni P, Girolami A (1996) Antiphospholipid antibodies, recurrent thromboembolism, and intensity of warfarin anticoagulation. Thromb Haemost 75:859

38. Rance A, Emmerich J, Fiessinger JN (1997) Anticardiolipin antibodies and recurrent thromboembolism. Thromb Haemost 77:221-222

39. Schulman S, Svenungsson E, Granqvist S (1998) Anticardiolipin antibodies predict early recurrence of thromboembolism and death among patients with venous thromboembolism following anticoagulant therapy. Duration of Anticoagulation Study Group. Am J Med 104:332-338

40. Crowther MA, Ginsberg JS, Julian J et al (2003) A comparison of two intensities of warfarin for the prevention of recurrent thrombosis in patients with the antiphospholipid antibody syndrome. $\mathrm{N}$ Engl J Med 349:1133-1138

41. Finazzi G, Marchioli R, Brancaccio V et al (2005) A randomized clinical trial of high-intensity warfarin vs. conventional antithrombotic therapy for the prevention of recurrent thrombosis in patients with the antiphospholipid syndrome (WAPS). J Thromb Haemost 3:848-853 
42. Ho WK, Hankey GJ, Quinlan DJ et al (2006) Risk of recurrent venous thromboembolism in patients with common thrombophilia: a systematic review. Arch Intern Med 166:729-736

43. Hron G, Eichinger S, Weltermann A et al (2006) Family history for venous thromboembolism and the risk for recurrence. Am J Med 119:50-53

44. Auerbach AD, Sanders GD, Hambleton J (2004) Cost-effectiveness of testing for hypercoagulability and effects on treatment strategies in patients with deep vein thrombosis. Am J Med 116:816-828

45. Straczek C, Alhenc-Gelas M, Aubry ML et al (2005) Genetic variation at the estrogen receptor alpha locus in relation to venous thromboembolism risk among postmenopausal women. J Thromb Haemost 3:1535-1537

46. Straczek C, Oger E, Yon de Jonage-Canonico MB et al (2005) Prothrombotic mutations, hormone therapy, and venous thromboembolism among postmenopausal women: impact of the route of estrogen administration. Circulation 112:3495-3500

47. Chopra N, Koren S, Greer WL et al (2002) Factor V Leiden, prothrombin gene mutation, and thrombosis risk in patients with antiphospholipid antibodies. J Rheumatol 29:1683-1688

48. Galli M, Finazzi G, Duca F et al (2000) The G1691 $\rightarrow$ A mutation of factor $\mathrm{V}$, but not the $\mathrm{G} 2 \mathrm{O2} 10 \rightarrow$ A mutation of factor II or the $\mathrm{C} 677 \rightarrow \mathrm{T}$ mutation of methylenetetrahydrofolate reductase genes, is associated with venous thrombosis in patients with lupus anticoagulants. Br J Haematol 108:865-870

49. Galli M, Borrelli G, Jacobsen EM et al (2007) Clinical significance of different antiphospholipid antibodies in the WAPS (warfarin in the antiphospholipid syndrome) study. Blood 110:1178-1183

50. Martínez-Berriotxoa A, Ruiz-Irastorza G, Egurbide MV et al (2007) Transiently positive anticardiolipin antibodies and risk of thrombosis in patients with systemic lupus erythematosus. Lupus 16:810-816

51. Espinosa G, Bucciarelli S, Tàssies D et al (2007) Persistently positive antiphospholipid antibodies are related with the appearance of thrombosis during follow-up of patients with antiphospholipid syndrome (abstract). Arthritis Rheum 56:s554

52. Cervera R, Khamashta MA, Shoenfeld Y et al (2009) Morbidity and mortality in the antiphospholipid syndrome during a 5-year period: a multicenter prospective study of 1,000 patients. Ann Rheum Dis 68:1428-1432

53. Sorice M, Griggi T, Circella A et al (1994) Detection of antiphospholipid antibodies by immunostaining on thin layer chromatography plates. J Immunol Methods 173:49-54

54. Erkan D, Derksen WJ, Kaplan V et al (2005) Real world experience with antiphospholipid antibody tests: how stable are results over time? Ann Rheum Dis 64:1321-1325

55. Rodríguez-Fernández S, Saavedra Ramírez P, Espinosa Garriga G et al (2009) Suspensión de la anticoagulación en pacientes con síndrome antifosfolipídico primario en los que se negativizan los anticuerpos antifosfolipídicos. Rev Clin Esp 209:357-358

56. Silver RK, MacGregor SN, Sholl JS et al (1993) Comparative trial of prednisone plus aspirin versus aspirin alone in the treatment of anticardiolipin antibody-positive obstetric patients. Am J Obstet Gynecol 169:1411-1417

57. Balasch J, Carmona F, Lopez-Soto A et al (1993) Low-dose aspirin for prevention of pregnancy losses in women with primary antiphospholipid syndrome. Hum Reprod 8:2234-2239

58. Carmona F, Font J, Azulay M et al (2001) Risk factors associated with fetal losses in treated antiphospholipid syndrome pregnancies: a multivariate analysis. Am J Reprod Immunol 46:274-279

59. Lima F, Khamashta MA, Buchanan NM et al (1996) A study of sixty pregnancies in patients with the antiphospholipid syndrome. Clin Exp Rheumatol 14:131-136

60. Granger KA, Farquharson RG (1997) Obstetric outcome in antiphospholipid syndrome. Lupus 6:509-513
61. Huong DL, Wechsler B, Bletry O et al (2001) A study of 75 pregnancies in patients with antiphospholipid syndrome. J Rheumatol 28:2025-2030

62. Silveira LH, Hubble CL, Jara LJ et al (1992) Prevention of anticardiolipin antibody-related pregnancy losses with prednisone and aspirin. Am J Med 93:403-411

63. Ruiz-Irastorza G, Khamashta MA, Hughes GR (2001) Antiaggregant and anticoagulant therapy in systemic lupus erythematosus and Hughes' syndrome. Lupus 10:241-245

64. Rosove MH, Tabsh K, Wasserstrum N et al (1990) Heparin therapy for pregnant women with lupus anticoagulant or anticardiolipin antibodies. Obstet Gynecol 75:630-634

65. Bates SM, Greer IA, Pabinger I et al (2008) Venous thromboembolism, thrombophilia, antithrombotic therapy, and pregnancy: American College of Chest Physicians Evidence-Based Clinical Practice Guidelines (8th Edition). Chest 133:844S-886S

66. Kutteh WH, Rote NS, Silver R (1999) Antiphospholipid antibodies and reproduction: the antiphospholipid antibody syndrome. Am J Reprod Immunol 41:133-152

67. ACOG Committee on Practice Bulletins-Obstetrics (2005) ACOG Practice Bulletin \#68: Antiphospholipid syndrome. Obstet Gynecol 106:1113-1121

68. Derksen RH, De Groot PG, Nieuwenhuis HK et al (2001) How to treat women with antiphospholipid antibodies in pregnancy? Ann Rheum Dis 60:1-3

69. Myones BL, McCurdy D (2000) The antiphospholipid syndrome: immunologic and clinical aspects. Clinical spectrum and treatment. J Rheumatol 58 (Suppl):20-28

70. Empson MB, Lassere M, Craig JC et al (2005) Prevention of recurrent miscarriage for women with antiphospholipid antibody or lupus anticoagulant. Cochrane Database of Systematic Reviews, Issue 2. Art. no. CD002859

71. Rai R, Cohen H, Dave M et al (1997) Randomised controlled trial of aspirin and aspirin plus heparin in pregnant women with recurrent miscarriage associated with phospholipid antibodies (or antiphospholipid antibodies). BMJ 314:253-257

72. Kutteh WH (1996) Antiphospholipid antibody-associated recurrent pregnancy loss: treatment with heparin and low-dose aspirin is superior to low-dose aspirin alone. Am J Obstet Gynecol 174:1584-1589

73. Farquharson RG, Quenby S, Greaves M (2002) Antiphospholipid syndrome in pregnancy: a randomized, controlled trial of treatment. Obstet Gynecol 100:408-413

74. Balasch J, Font J, Lopez-Soto A et al (1990) Antiphospholipid antibodies in unselected patients with repeated abortion. Hum Reprod 5:43-46

75. Pattison NS, Chamley LW, Birdsall M et al (2000) Does aspirin have a role in improving pregnancy outcome for women with the antiphospholipid syndrome? A randomized controlled trial. Am J Obstet Gynecol 183:1008-1012

76. Cowchock S, Reece EA (1997) Do low-risk pregnant women with antiphospholipid antibodies need to be treated? Organizing Group of the Antiphospholipid Antibody Treatment Trial. Am J Obstet Gynecol 176:1099-1100

77. Asherson RA, Cervera R, Piette JC et al (1998) Catastrophic antiphospholipid syndrome. Clinical and laboratory features of 50 patients. Medicine (Baltimore) 77:195-207

78. Asherson RA, Cervera R, Piette JC et al (2001) Catastrophic antiphospholipid syndrome: clues to the pathogenesis from a series of 80 patients. Medicine (Baltimore) 80:355-377

79. Bayraktar UD, Erkan D, Bucciarelli S et al (2007) The clinical spectrum of catastrophic antiphospholipid syndrome in the absence and presence of lupus. J Rheumatol 34:346-352

80. Asherson RA, Cervera R, de Groot PG et al (2003) Catastrophic antiphospholipid syndrome: international consensus statement on classification criteria and treatment guidelines. Lupus 12:530-534 